\title{
Clima organizacional y estrés en los alumnos de educación secundaria de una I. E. PNP
}

\author{
Organizational climate and stress in secondary education students \\ of the PNP I.E.
}

\section{Clima organizacional e estresse em alunos do ensino médio de um I.E. PNP}

\section{ARTÍCULO DE INVESTIGACIÓN}

\author{
Gregorio Ernesto Tomás Quispe \\ gtomas@ucvvirtual.edu.pe \\ https://orcid.org/0000-0002-4124-5878 \\ Universidad César Vallejo, Piura-Perú
}

\author{
Orlando Mamani Rodríguez \\ orlandomamanir@hotmail.com \\ https://orcid.org/0000-0002-4163-8102
}

Ministerio de Educación, Lima, Perú

\section{RESUMEN}

\begin{abstract}
Esta investigación tiene como objetivo, determinar la relación entre el clima organizacional y estrés en los alumnos del $5^{\circ}$ año de educación secundaria de la I. E. PNP José Héctor Rodríguez Trigoso-San Martin de Porres, la metodología empleada se basa en un diseño no experimental de campo, con un nivel descriptivo correlacional de corte transversal sobre una muestra de 112 alumnos. Como conclusión se indica que en base a los estudios obtenidos, se aprecia que el clima organizacional y el estrés están estrechamente relacionados. Es importante que todos los involucrados estén dispuestos a enfrentar los cambios ocurridos dentro de esta nueva normalidad y poder aprender a confrontar situaciones que generen presión en el estudiante y así puedan controlar sus emociones sin llegar al límite de sus capacidades, convirtiendo estas situaciones en estrés que a corto o largo plazo pueda generarles daños en su salud tanto física como mental.
\end{abstract}

Palabras clave: Clima organizacional; estrés académico; alumnos

\section{ABSTRACT}

This research aims to determine the relationship between organizational climate and stress in students in the 5th year of secondary education of the IE PNP José Héctor Rodríguez Trigoso-San Martin de Porres, the methodology used is based on a non-experimental field design, with a cross-sectional correlational descriptive level on a sample of 112 students. As a conclusion it is indicated that based on the studies obtained, it is appreciated that organizational climate and stress are closely related. It is important that all those involved are willing to face the changes that have occurred within this new normal and to be able to learn to confront situations that generate pressure on the student and thus be able to control their emotions without reaching the limit of their capacities, turning these situations into stress that In the short or long term, it can damage their physical and mental health.

Key words: Organizational climate; academic stress; students

\section{RESUMO}

Esta pesquisa visa determinar a relação entre clima organizacional e estresse em alunos do $5^{\circ}$ ano do ensino médio do IE PNP José Héctor Rodríguez Trigoso-San Martin de Porres, a metodologia utilizada é baseada em um desenho não experimental de campo, com um nível descritivo correlacional transversal em uma amostra de 112 alunos. Como conclusão, indica-se que com base nos estudos obtidos, percebe-se que o clima organizacional e o estresse estão intimamente relacionados. É importante que todos os envolvidos estejam dispostos a enfrentar as mudanças ocorridas dentro dessa nova normalidade e possam aprender a enfrentar as situações que geram pressão no aluno e assim conseguir controlar suas emoções sem atingir o limite de suas capacidades, transformando essas situações em estresse que, a curto ou longo prazo, pode prejudicar sua saúde física e mental.

Palavras-chave: Clima organizacional; estresse acadêmico; alunos 


\section{INTRODUCCCIÓN}

Todas las organizaciones comparten ciertos elementos propios del proceso administrativo, sin embargo cada empresa posee elementos y características que lo hacen diferente de su competencia, una de estas propiedades es la habilidad que posee los trabajadores para el manejo de situaciones y el desenvolvimiento de sus actividades, es allí donde influye directamente el clima organizacional.

El clima organizacional está relacionado con la forma en que los trabajadores perciben a la organización y la manera en que se desenvuelven en sus actividades particulares. Es por ello, que el clima organizacional puede ser de gran confianza y progreso así como también puede mostrar aspectos como inseguridad o miedo, y conocer como es percibido el clima dentro de la empresa es una poderosa herramienta para conocer si existen fallas $o$ al contrario mantener reforzado los procesos administrativos que le permita un excelente bienestar a la empresa.

Los autores, Cisneros y Paredes (2013), menciona que la primera vez que se incluyó el término clima organizacional en la psicología organizacional fue en los años 1960 por Gellerman, sin embargo no se definía claramente el término, llegando a ser confundido con la cultura organizacional, o con el liderazgo. Es importante resaltar que el clima organizacional no debe ser considerada como una norma, sino que esta es resultado de una mezcla de diversos aspectos que relacionan entre si y que son influyentes en la actuación diaria de todo el personal que hace vida dentro de la organización.

En este sentido Brune, 1999, Chiavenato, 2000 y Valencia, 2004 citados por Cisneros y Paredes (2013) mencionan que al clima organizacional como:

El resultado de la interrelación de
las diversas partes de una
organización, de tal manera, que
el comportamiento de un
trabajador depende los factores
organizacionales existentes y de
las percepciones que tenga de
estos factores (p. 44).

Existen diversas maneras de poder conocer el clima organizacional de una empresa, García y Bedoya 1997 citado por Segredo P (2013), expresan que se puede aplicar la observación directa ya que por medio de esta técnica se puede visualizar de forma directa como es el comportamiento de los trabajadores y su desenvolvimiento en las activdades que le correspondan, igualmente se pueden realizar entrevistar y custionarios diseñados para este fin.

De la misma manera existen diversos tipos de clima organizacional y para Loaiza, Salazar, Espinoza, y Lozano (2019), son las dispuestas en la Tabla 1. 
Tabla 1. Tipos de Clima Organizacional según diversas teorías.

\section{CLASIFICACIÓN}

TIPOS DE CLIMA ORGANIZACIONAL

Teoria de Lewin, Lippit y White (1839) Faire, en cada uno de ellos ejerce una influencia distinta sobre los comportamientos individuales y grupales.

Teoria de Litwin y Strunger (1968)

Rensis Likert (1967)
Estudia tres estilos distintos de liderazgo: democrático, autocrático y otro orientado al logro, cada uno de ellos genera un tipo específico de clima.

Considera los tipos de clima asociado a la figura del líder y el tipo de interacción, así lo divide en: Clima autoritario, Clima paternalista, Clima consultivo y Clima participativo.

Fuente: (Loaiza, Salazar, Espinoza, y Lozano, 2019).

Es importante entender que el clima organizacional no es igual que cultura organizacional, si bien ambas se relacionan entre si, cada una cumple una funcion especifica dentro de la organización. Williams, (2013) menciona que el clima organizacional permite poder verificar como es la cultura laboral dentro de la organización mientras que la cultura son las condiciones por medio del cual se desarrollan las interacciones entre los trabajadores $\mathrm{y}$ esto crea el clima organizacional.

De una manera mas clara se presente una comparación entre ambas definiciones realizada por Denison, (1996) citado por Williams (2013) y se presenta en la Tabla 2.

Tabla 2. Comparacion entre cultura organizacional y clima organizacional.

\begin{tabular}{lll}
\hline \multicolumn{1}{c}{ DIFERENCIAS } & \multicolumn{1}{c}{ CLIMA ORGANIZACIONA } & \multicolumn{1}{c}{\begin{tabular}{c}
\multicolumn{1}{c}{ CULTURA } \\
ORGANIZACIONAL
\end{tabular}} \\
\hline $\begin{array}{l}\text { Epistemología } \\
\text { Metodología }\end{array}$ & Contextualizacion/Ideográfica & Comparatica/Nomotética \\
Nivel de análisis & Cuelitativa/Observación de Campo & Cuantitativa/encuesta \\
Orientación Temporal & Valores y presunciones subyacentes & Consenso sobre percepciones \\
Fundamentación Teórica & Evolución Hostórica & Construccionismos social/Teoría Crítica \\
Disciplina & Sociología/antropología & $\begin{array}{l}\text { Perspectiva/No lewiniana } \\
\text { Psicología }\end{array}$ \\
\hline
\end{tabular}

Fuente: (Williams, 2013).

En este sentido el clima organizacional permite comprender como actúan y se desenvuelven los trabajadores dentro de ella frente a las situaciones diarias, frente a la rutina, las normas y exigencias establecidas por los directivos, de manera que se pueda entender si tienen agrado o desagrado a estas situaciones.

El clima organizacional surge en todos los niveles organizativos, de los cuales las instituciones formativas no escapan de esta realidad, ya que sirve de filtro para conocer los 
elementos que la conforman, en cuanto a su estructura, el liderazgo, la toma de decisión, entre otros, y permite determinar cómo estos aspectos influyen en el desenvolvimiento de los alumnos que hacen vida dentro de las instituciones.

Actualmente producto de la Pandemia alrededor del mundo, han surgido una serie de normas restrictivas en cuanto a la movilización social que no han permitido el retorno a las aulas de clases, lo que afecta en gran medida las actividades de interacción humana restringiendo esto a lo más mínimo posible. Las instituciones educativas se han visto obligadas a cambiar como sistema, y las clases impartidas han cambiado de un salón de clases a una pantalla de computadora o dispositivo móvil donde el estudiante debe seguir los procesos educativos y poder cumplir los objetivos propuestos. Como el alumno está acostumbrado a relacionarse directamente con los docentes debe adaptarse a las nuevas herramientas electrónicas y las plataformas que los colegios implementan para continuar con sus actividades, e inclusive sus padres o representantes en el hogar deben adaptarse a este cambio forjando y creando horarios de estudio, que permitan mantener cierta normalidad en el desarrollo de sus actividades educativas.

Asimismo los docentes deben procurar mantener una interacción con los estudiantes asegurando la participación de estos en las actividades propuestas a través de estas plataformas, con la desventaja de que no pueden controlar este aspecto, es decir que en estas circunstancias las formas de interacción en la gestión educativa han cambiado en gran medida.

En este sentido, en ocasiones los involucrados no se sienten cómodos en sus áreas de trabajo y surgen incomodidades e inseguridades que en ocasiones provocan estrés en las personas. El estrés es un desequilibrio que sufren las personas cuando superan el límite que poseen para lidiar con situaciones laborales, educativas o personales que repercuten en su quehacer diario y puede llegar a afectar la salud del individuo, donde el organismo responde $\mathrm{y}$ puede generar malestares físicos en la persona.

Moreno y Báez 2010 citado por Osorio y Cárdenas (2017), definen el estrés laboral como:

El resultado de la aparición de factores psicosociales de riesgo, o como riesgo psicosocial que por sí mismo tiene "alta probabilidad de afectar de forma importante a la salud de los trabajadores y al funcionamiento empresarial a medio y largo plazo (p. 82).

Quito, Tamayo, Buñay, y Neira (2017) mencionan que las personas deben controlar sus emociones y con ello el estrés para evitar consecuencias que puedan perjudicar su estado de salud ficsica y mental, ya que el estrés puede generar trastornos personales, problemas con la sociedad y con la familia.

El estrés academico es producto de diversos factores incluyendo las constantes obligaciones y responsabilidades que deben cumplir los estudiantes diariamente causandole en ocasiones una sobrecarga de actividades, aunado a esto la presion que ejercen sus familiares, profesores e inclusive los compañeros rovocan un gran peso sobre el estudiante que en ocasiones no sabe como lidiar con estas situaciones.

Barraza, 2006 citado por Quito et al. (2017) señala que: 
El estrés académico es un proceso complejo de carácter psicológico y adaptativo, que se pone en manifiesto al momento que el alumno lleva a cabo su propia evaluacion de las demandas del contexto escolar, clasificandolos como estresores input, cuando los estresores generan una situación estresante que provoca desequilibrio desencadena una serie de sintomas denominados indicadores de desequilibrio; y por ultimo el alumno se ve obligado a tomar acciones de afrontamiento para restaurar su equilibrio.

Se puede mencionar que, si un estudiante está inmerso en un clima organizacional caótico también desarrollará mecanismos para manejar esta condición, que podría estar afectándolo conductual y actitudinalmente, por ello es necesario que las estrategias utilizadas por las instituciones educativas deben encaminarse de manera sistemática, planificada y coherente acorde a la realidad e idiosincrasia del lugar, también las formas de comunicación y trato con todo el personal de la comunidad educativa, de tal manera, que los actores educativos se sientan comprometidos con llevar adelante y alcanzar la visión y misión de la Institución Educativa.

Como resultante de este análisis se plantea el siguiente problema: ¿Cuál es la relación entre el clima organizacional y estrés en los alumnos del $5^{\circ}$ año de educación secundaria de la I. E. PNP José Héctor Rodríguez TrigosoSan Martin de Porres?

Al realizar la investigación, se plantea como hipótesis la existencia de una relación entre clima organizacional y estrés en los alumnos del $5^{\circ}$ año de educación secundaria de la I. E. PNP José Héctor Rodríguez Trigoso-
San Martin de Porres.

Asimismo, se propone que existe relación entre clima organizacional y los estresores, las reacciones físicas, las reacciones psicológicas, las reacciones comportamentales $y$ las estrategias de afrontamiento en los alumnos del $5^{\circ}$ año de educación secundaria de la I. E. PNP José Héctor Rodríguez Trigoso-San Martin de Porres.

\section{METODOLOGÍA}

Se trató de un estudio campo, con un nivel descriptivo correlacional de corte transversal; porque tiene como propósito saber cómo se pueden comportar un concepto o variable conociendo el comportamiento de otras variables relacionadas Hernández, Fernández, y Baptista (2010), intentan medir o recoger información de manera independiente o conjunta sobre las variables a los que se refieren; y se recolectan datos en un solo momento, en un tiempo único.

Palella Stracuzzi y Martins Pestana (2010), definen la población como el conjunto de unidades de las que se desea obtener información y sobre las que se van a generar conclusiones. En este sentido la población de esta investigación estuvo conformada por 718 estudiantes del $1^{\circ}$ al $5^{\circ}$ año de educación secundaria de la I. E. PNP José Héctor Rodríguez Trigoso-San Martin de Porres en Perú.

De la misma manera para seleccionar los elementos de la muestra se realizó través de la muestra censal compuesta por 112 alumnos, de tres aulas del $5^{\circ}$ año de secundaria donde se realizaban acciones de tutoría, estas secciones son $5^{\circ} \mathrm{A}, 5^{\circ} \mathrm{B}$ y $5^{\circ} \mathrm{C}$ de la I. E. PNP José Héctor Rodríguez Trigoso-San Martin de Porres (ver Tabla 3), lo que facilitó la toma de datos, por tener contacto directo con los alumnos. 
Tabla 3. Frecuencia de los alumnos que participaron en la toma de datos por aula.

\begin{tabular}{cc}
\hline GRADO/ESTUDIO & NÚMERO DE ALUMNOS \\
\hline $\mathbf{5}^{\circ} \mathbf{A}$ & 40 \\
$\mathbf{5}^{\circ} \mathbf{B}$ & 40 \\
$\mathbf{5}^{\circ} \mathbf{C}$ & 32 \\
\hline TOTAL & 112 \\
\hline
\end{tabular}

La técnica de recolección de datos que se utilizó en este trabajo investigativo fue mediante el análisis descriptivo y la técnica fue la encuesta, y el instrumento fue el cuestionario el cual tuvo como objetivo recopilar información para determinar, la relación del entre las variables clima organizacional y estrés.

El instrumento estuvo compuesto por la dimensión Clima Organizacional, la cual se caracterizó en una ficha técnica de Quispe Ayllón, y Nonones Alcalá.

\section{Significación}

El Cuestionario de Clima Organizacional contenía nueve dimensiones, que evaluaban las percepciones de los alumnos de la Institución Educativa. La dimensión (I) constó de cuatro indicadores de estructura, (II) cinco indicadores de recompensa, (III) cuatro indicadores de relaciones, (IV) cuatro indicadores de identidad, (V) tres indicadores de cooperación, (VI) cuatro indicadores de responsabilidad, (VII) tres indicadores de conflictos, (VIII) dos indicadores de estándares y (IX) un indicador de desafío.

Los puntajes para los ítems implicaban una alta o baja frecuencia, de acuerdo a como se percibía el clima organizacional. (Tabla 4).

Tabla 4. Puntajes de los Ítems.

\begin{tabular}{lccccc}
\hline & $\begin{array}{c}\text { EN TOTAL } \\
\text { ACUERDO }\end{array}$ & $\begin{array}{c}\text { DE } \\
\text { ACUERDO }\end{array}$ & INDIFERENTE & $\begin{array}{c}\text { EN } \\
\text { DESACUERDO }\end{array}$ & $\begin{array}{c}\text { TOTAL } \\
\text { DESACUERDO }\end{array}$ \\
\hline Ítems Positivos & 5 & 4 & 3 & 2 & 1 \\
Ítems Negativos & 5 & 2 & 3 & 4 & 5 \\
\hline
\end{tabular}

Para determinar si el clima organizacional es malo, aceptable o adecuado se realizó de la siguiente forma: entre el puntaje mínimo y el máximo posible (30 y 150) se establecen 3 intervalos de igual tamaño dividiendo la diferencia de los dos puntajes entre 3 y a partir del puntaje mínimo se suma el resultado obtenido así: a). Clima Organizacional Malo: De 30 a 70 puntos; b). Clima Organizacional Aceptable: De 71 a 110 puntos, y c). Clima Organizacional Adecuado: entre 111 y 150 puntos.

Para la validez de instrumento se tomó en cuenta la validez de contenido mediante un juicio de expertos conformado por tres jueces quienes, mediante criterios preestablecidos determinaron su validez para la medición de la variable 1 . El puntaje obtenido mediante el criterio de expertos fue de 90 lo cual indica que el instrumento es válido y aplicable para medir el clima organizacional. 
En cuanto a la confiabilidad del instrumento esta fue determinada mediante el análisis del coeficiente alfa de Cronbach. Se aplicó a una muestra piloto de 30 alumnos; obteniendo los resultados reflejados en la Tabla 5 .

Tabla 5. Confiabilidad del cuestionario de clima organizacional.

\begin{tabular}{llcc}
\hline \multicolumn{4}{c}{ RESUMEN DEL PROCESAMIENTO DE LOS CASOS } \\
\hline \multirow{4}{*}{ Casos } & Válidos & $\mathbf{N}$ & $\%$ \\
& Excluidos ${ }^{a}$ & 30 & 100 \\
& Total & 0 & 0.0 \\
& Alfa de Cronbach & 30 & 100 \\
& . Estadísticos de fiabilidad & N de elementos \\
& 924 & 9 & \\
\hline
\end{tabular}

Mediante el índice alfa de Cronbach, 0.924 , fue el resultado obtenido para los 9 elementos que conformaba la escala de clima organizacional aplicada, en este sentido se indica que el puntaje indicado señala que el instrumento tuvo una alta confiabilidad estadística.

En cuanto a la variable 2 Estrés esta se caracterizó en: Ficha Técnica; nombre: inventario del estrés, Autor: Dr. Arturo Barraza Macías, Profesor Investigador de la Universidad Pedagógica de Durango y Coordinador del Grupo de Investigación "El Estrés en los Agentes Educativos".

\section{Características}

El Inventario del Estrés, se encuentra conformado por 31 ítems distribuidos de la siguiente manera:

Un ítem de filtro que, en términos dicotómicos (si-no), permite determinar si el encuestado es candidato o no a contestar el inventario, un ítem que, en un escalamiento tipo Lickert de cinco valores numéricos (del 1 al 5, donde uno es poco y cinco mucho), permite identificar el nivel de intensidad del estrés, Ocho ítems que, en un escalamiento tipo Lickert de cinco valores categoriales (nunca, rara vez, algunas veces, casi siempre y siempre), permiten identificar la frecuencia en que las demandas de evaluación del entorno son valoradas como estímulos estresores, 15 ítems que, en un escalamiento tipo Lickert de cinco valores categoriales (nunca, rara vez, algunas veces, casi siempre y siempre), permiten identificar la frecuencia con que se presentan los síntomas o reacciones al estímulo estresor, seis ítems que, en un escalamiento tipo Lickert de cinco valores categoriales (nunca, rara vez, algunas veces, casi siempre y siempre), permiten identificar la frecuencia de uso de las estrategias de afrontamientos.

\section{Validez}

De los diferentes tipos de evidencia que se pueden recolectar para establecer la validez del proceso de medición de un instrumento, en el caso del Inventario del Estrés de Examen se recolectó evidencia con relación a la estructura interna. Las estrategias utilizadas fueron: el análisis factorial, el análisis de consistencia interna y el análisis de grupos contrastados. 


\section{Confiabilidad del Instrumento}

Para determinar el grado de confiabilidad del instrumento se aplicó a una muestra piloto de 30 alumnos; obteniéndose los resultados mostrados en la Tabla 5.

Tabla 5. Confiabilidad del estrés.

\begin{tabular}{cccc}
\hline \multicolumn{4}{c}{ Resumen del procesamiento de los casos } \\
\hline & Válidos & $\mathbf{N}$ & $\%$ \\
\hline \multirow{2}{*}{ Casos } & Excluidos & 112 & 100 \\
& Total & 0 & 0.0 \\
a. Eliminación por lista basada en todas las variables del procedimiento. & \\
& Estadísticos de fiabilidad & $\mathbf{1 0 0}$ \\
Alfa de Cronbach & N de elementos \\
& .877 & 7 & \\
\hline
\end{tabular}

Mediante el índice alfa de Cronbach, 0.877 obtenido para los 7 elementos que conformaron la escala de estrés aplicada, en donde se señalar que el puntaje indicado señala que el instrumento tiene una buena confiabilidad estadística; por lo cual se espera que los puntajes en una muestra mayor puedan ser similares a los establecidos.

\section{Prueba de confiabilidad del instrumento}

Se muestran los resultados de la prueba de confiabilidad realizada en la Tabla 6 .

Tabla 6. Prueba de confiabilidad de la variable estrés.

\begin{tabular}{cc}
\hline \multicolumn{2}{c}{ Estadísticos de fiabilidad } \\
\hline Alfa de Cronbach & $\mathrm{N}$ de elementos \\
, 867 & 31 \\
\hline
\end{tabular}

El valor del alfa de Cronbach 0,867 muestra que los ítems del instrumento de la variable estrés es altamente confiable según se destacó en la Tabla 6.
Asimismo, se realiza la prueba de confiabilidad de la variable clima organizacional obteniendo los resultados mostrados en la Tabla 7.

Tabla 7. Prueba de confiabilidad de la variable clima organizacional.

\section{Estadísticos de fiabilidad}

Alfa de Cronbach

$\mathrm{N}$ de elementos

,935

30

El valor del alfa de Cronbach 0,935 muestra que los ítems de la variable clima organizacional son altamente confiables. 


\section{RESULTADOS Y DISCUSIÓN}

Posterior a la aplicación de la encuesta a la muestra elegida se procedió al análisis de la información recolectada a través del programa estadístico SPSS versión 21.0 en español para obtener los resultados pertinentes al estudio, los cuales se muestran mediante tablas, con su correspondiente interpretación.

En la Tabla 8 se muestra la descripción de los resultados de la variable clima organizacional de la siguiente manera:

Tabla 8. Distribución de frecuencias y porcentajes del clima organizacional de los estudiantes del $5^{\circ}$ año de educación secundaria de la IE PNP José Héctor Rodríguez Trigoso-San Martin de Porres.

\begin{tabular}{cccccc}
\hline & \multicolumn{3}{c}{ Clima organizacional } \\
& Frecuencia & Porcentaje & $\begin{array}{c}\text { Porcentaje } \\
\text { válido }\end{array}$ & $\begin{array}{c}\text { Porcentaje } \\
\text { acumulado }\end{array}$ \\
\hline \multirow{3}{*}{ Válidos } & Malo & 17 & 15,2 & 15,2 & 15,2 \\
& Regular & 64 & 57,1 & 57,1 & 72,3 \\
& Bueno & 31 & 27,7 & 27,7 & 100,0 \\
\hline & Total & $\mathbf{1 1 2}$ & $\mathbf{1 0 0 , 0}$ & $\mathbf{1 0 0 , 0}$ & \\
\hline
\end{tabular}

De la Tabla 8 se puede observar que, el 15,2\% de los estudiantes del $5^{\circ}$ año de educación secundaria de la IE PNP José Héctor Rodríguez Trigoso-San Martin de Porres, manifestaron que el clima organizacional es malo, el 57,1\% regular y el 27,7 \% bueno.

De la misma manera en la Tabla 9 se muestra la descripción de los resultados de la variable estrés.

Tabla 9. Distribución de frecuencias y porcentajes del estrés de los estudiantes del $5^{\circ}$ año de educación secundaria de la IE PNP José Héctor Rodríguez Trigoso-San Martin de Porres.

\begin{tabular}{cccccc}
\hline & & ESTRÉS & & \\
& Frecuencia & Porcentaje & Porcentaje válido & $\begin{array}{c}\text { Porcentaje } \\
\text { acumulado }\end{array}$ \\
\hline \multirow{3}{*}{ Válidos } & Leve & 36 & 32,1 & 32,1 & 32,1 \\
& Moderado & 71 & 63,4 & 63,4 & 95,5 \\
& Profundo & 5 & 4,5 & 4,5 & 100,0 \\
\hline & Total & $\mathbf{1 1 2}$ & $\mathbf{1 0 0 , 0}$ & $\mathbf{1 0 0 , 0}$ & \\
\hline
\end{tabular}

Se apreció en la Tabla 9 que, el 32,1 \% de los estudiantes del $5^{\circ}$ año de educación secundaria de la IE PNP José Héctor Rodríguez Trigoso-San Martin de Porres. Tienen estrés leve, el 63,4 \% moderado y el $4,5 \%$ profundo.

Seguidamente, en la Tabla 10 se muestra la descripción de los resultados de las dimensiones de la variable estrés. 
Tabla 10. Distribución de frecuencias y porcentajes según nivel de estrés por dimensiones de los estudiantes del $5^{\circ}$ año de educación secundaria de la IE PNP José Héctor Rodríguez TrigosoSan Martin de Porres.

\begin{tabular}{lcccccccccc}
\hline & \multicolumn{2}{c}{ ESTRESORES } & \multicolumn{2}{c}{ REAC. } & \multicolumn{2}{c}{ REAC. } & \multicolumn{2}{c}{ RES. COMPOR. } & \multicolumn{2}{c}{ ESTRAT. } \\
& $\mathbf{f i}$ & $\mathbf{\%}$ & $\mathbf{f i}$ & $\mathbf{\%}$ & $\mathbf{f i}$ & $\mathbf{\%}$ & $\mathbf{f i}$ & $\mathbf{\%}$ & fi & $\mathbf{\%}$ \\
& 62 & $\mathbf{5 5 , 4}$ & 18 & $\mathbf{1 6 , 1}$ & 44 & $\mathbf{3 9 , 3}$ & 21 & $\mathbf{1 8 , 8}$ & 13 & $\mathbf{1 1 , 6}$ \\
& 49 & $\mathbf{4 3 , 8}$ & 85 & $\mathbf{7 5 , 9}$ & 60 & $\mathbf{5 3 , 6}$ & 56 & $\mathbf{5 0 , 0}$ & 89 & $\mathbf{7 9 , 5}$ \\
Moderado & 1 & $\mathbf{9}$ & 9 & $\mathbf{8 , 0}$ & 8 & $\mathbf{7 , 1}$ & 35 & $\mathbf{3 1 , 3}$ & 10 & $\mathbf{8 , 9}$ \\
Profundo & 112 & $\mathbf{1 0 0 , 0}$ & 112 & $\mathbf{1 0 0 , 0}$ & 112 & $\mathbf{1 0 0 , 0}$ & 112 & $\mathbf{1 0 0 , 0}$ & 112 & $\mathbf{1 0 0 , 0}$ \\
\hline Total & & & & & & & & & &
\end{tabular}

En la Tabla 10 se observó que en los resultados por cada dimensión existió un grupo del $55.4 \%$ de estudiantes en la dimensión estresores se encontró en el nivel leve, el $43,8 \%$ en moderado y el $0,9 \%$ en nivel profundo, en la dimensión reacciones físicas el $16,1 \%$ de estudiantes se encontró en un nivel leve, el 75,9 \% moderado y el $8 \%$ profundo, en la dimensión reacciones psicológicas el 39,3\% se encontró en el nivel leve, el 53,6\% moderado y el 7,1 \% profundo, en la dimensión reacciones comportamentales, el 18,8\% se encontró en el nivel leve, el $50 \%$ moderado y el 31,3 \% profundo y en la dimensión estrategias de afrontamiento el
11,6 \% se encontró en el nivel leve, el 79,5 \% moderado y el 8,9\% profundo.

\section{Prueba de Hipótesis General}

Ho No existe correlación significativa entre clima organizacional y estrés en alumnos del $5^{\circ}$ año de educación secundaria de la I. E. PNP José Héctor Rodríguez TrigosoSan Martin de Porres.

H1 Existe correlación significativa entre clima organizacional y estrés en alumnos del $5^{\circ}$ año de educación secundaria de la I. E. PNP José Héctor Rodríguez TrigosoSan Martin de Porres.

Tabla 11. Coeficiente de correlación de Spearman de la variable clima organizacional y el estrés.

\begin{tabular}{cccc}
\hline & & Estrés \\
\hline Rho de & Clima organizacional & Coeficiente de correlación &,$- 367^{* *}$ \\
Spearman & Sig. (bilateral) &, 000 \\
& $\mathrm{~N}$ & 112 \\
\hline
\end{tabular}

**. La correlación es significativa al nivel 0,01 (bilateral).

Los resultados del análisis estadístico en la Tabla 11 dan cuenta de la existencia de una relación inversa Rho de Spearman $\mathrm{r}=$ $0,367^{* *}$ entre las variables: clima organizacional y el estrés. Este grado de correlación indica que la relación entre las variables fue inversa mostrándose un nivel de correlación baja. En consecuencia se pudo afirmar que, existió relación negativa entre clima organizacional y estrés en alumnos del 
$5^{\circ}$ año de educación secundaria de la I. E. PNP José Héctor Rodríguez Trigoso-San Martin de Porres. $(r=-0,367)$.

\section{Decisión estadística}

La significación de $\mathrm{p}=0,000$ mostró que es menor a 0,5 lo que permite señalar que la relación es significativa por lo tanto se rechaza la hipótesis nula y se acepta la hipótesis alternativa, es decir que el clima organizacional tiene relación inversa $y$ significativa en el estrés de los alumnos del $5^{\circ}$ año de educación secundaria de la I. E. PNP José Héctor Rodríguez Trigoso-San Martin de Porres, Perú

\section{Prueba de Hipótesis específica 1}

Ho No existe correlación significativa entre clima organizacional y los estresores en alumnos del $5^{\circ}$ año de educación secundaria de la I. E. PNP José Héctor Rodríguez Trigoso-San Martin de Porres.

H1 Existe correlación significativa entre clima organizacional y los estresores en alumnos del $5^{\circ}$ año de educación secundaria de la I. E. PNP José Héctor Rodríguez Trigoso-San Martin de Porres.

Tabla 12. Coeficiente de correlación de Spearman de la variable clima organizacional y la dimensión estresores.

\begin{tabular}{|c|c|c|c|}
\hline & & & Estresores \\
\hline \multirow{3}{*}{ Rho de Spearman } & Clima organizacional & $\begin{array}{l}\text { Coeficiente de } \\
\text { correlación }\end{array}$ &,$- 340^{* *}$ \\
\hline & & Sig. (bilateral) & ,000 \\
\hline & & $\mathrm{N}$ & 112 \\
\hline
\end{tabular}

**. La correlación es significativa al nivel 0,01 (bilateral).

Los resultados del análisis estadístico en la Tabla 12 dieron cuenta de la existencia de una relación Rho de Spearman $r=-0,340^{* *}$ entre la variable: clima organizacional y la dimensión estresores. Este grado de correlación indicó que la relación entre las variables fue inversa y mostró una correlación baja. En consecuencia se puede afirmar que, existe relación inversa entre clima organizacional y los estresores en alumnos del $5^{\circ}$ año de educación secundaria de la I. E. PNP José Héctor Rodríguez TrigosoSan Martin de Porres, Perú.

\section{Decisión estadística}

La significación de $\mathrm{p}=0,000$ mostró que es menor a 0,5 lo que permitió señalar que la relación fue significativa, por lo tanto se rechaza la hipótesis nula y se acepta la hipótesis alternativa, es decir, que el clima organizacional tiene relación inversa y significativa en el estrés de los alumnos del $5^{\circ}$ año de educación secundaria de la I. E. PNP José Héctor Rodríguez Trigoso-San Martin de Porres. 


\section{Prueba de Hipótesis específica 2}

Ho No existe correlación significativa entre clima organizacional y las reacciones físicas en alumnos del $5^{\circ}$ año de educación secundaria de la I. E. PNP José Héctor Rodríguez Trigoso-San Martin de Porres.
H1 Existe correlación significativa entre clima organizacional y las reacciones físicas en alumnos del $5^{\circ}$ año de educación secundaria de la I. E. PNP José Héctor Rodríguez Trigoso-San Martin de Porres.

Tabla 13. Coeficiente de correlación de Spearman de la variable clima organizacional y la dimensión reacciones físicas.

\begin{tabular}{lccc}
\hline & & Reacciones Físicas \\
\hline \multirow{3}{*}{ Rho de Spearman } & Clima organizacional & Coeficiente de correlación &,$- 387^{* *}$ \\
& & Sig. (bilateral) &, 000 \\
& & N & 112 \\
\hline
\end{tabular}

**. La correlación es significativa al nivel 0,01 (bilateral).

Los resultados del análisis estadístico dan cuenta de la existencia de una relación Rho de Spearman $r=-0,387^{* *}$ entre la variable: clima organizacional y la dimensión reacciones físicas. Este grado de correlación indica que la relación entre las variables es inversa y tiene un nivel de correlación baja. En consecuencia se puede afirmar que, existe relación inversa entre clima organizacional y las reacciones físicas en alumnos del 5o año de educación secundaria de la I. E. PNP José Héctor Rodríguez Trigoso-San Martin de Porres.

\section{Decisión estadística}

La significación de $\mathrm{p}=0,000$ muestra que es menor a 0,5 lo que permite señalar que la relación es significativa por lo tanto se rechaza la hipótesis nula y se acepta la hipótesis alternativa, es decir que el clima organizacional tiene relación inversa y significativa en el estrés de los alumnos de educación secundaria de la I. E. PNP José Héctor Rodríguez Trigoso-San Martin de Porres.

\section{Prueba de Hipótesis específica 3}

Ho No existe correlación significativa entre clima organizacional y las reacciones psicológicas en alumnos del $5^{\circ}$ año de educación secundaria de la I. E. PNP José Héctor Rodríguez Trigoso-San Martin de Porres.

H1 Existe correlación significativa entre clima organizacional y las reacciones psicológicas en alumnos del $5^{\circ}$ año de educación secundaria de la I. E. PNP José Héctor Rodríguez Trigoso-San Martin de Porres. 
Tabla 14. Coeficiente de correlación de Spearman de la variable clima organizacional y la dimensión reacciones psicológicas.

\begin{tabular}{lccc}
\hline & & Reacciones Psicológicas \\
\hline \multirow{2}{*}{ Rho de Spearman } & Clima organizacional & $\begin{array}{c}\text { Coeficiente de } \\
\text { correlación }\end{array}$ &,$- 259^{* *}$ \\
& Sig. (bilateral) &, 006 \\
& $\mathrm{~N}$ & 112 \\
\hline
\end{tabular}

**. La correlación es significativa al nivel 0,01 (bilateral).

Los resultados del análisis estadístico en la Tabla 14 dieron cuenta de la existencia de una relación Rho de Spearman $r=-0,259^{* *}$ entre la variable: clima organizacional y la dimensión reacciones psicológicas. Este grado de correlación indicó que la relación entre las variables es inversa y tiene un nivel de correlación baja. En consecuencia se pudo afirmar que, existió relación inversa entre clima organizacional y las reacciones psicológicas en alumnos del $5^{\circ}$ año de secundaria de la I. E. PNP José Héctor Rodríguez Trigoso-San Martin de Porres.

\section{Decisión estadística}

La significación de $\mathrm{p}=0,006$ muestra que es menor a 0,5 lo que permite señalar que la relación es significativa por lo tanto se rechaza la hipótesis nula y se acepta la hipótesis alternativa, es decir que el clima organizacional tiene relación inversa y significativa en el estrés de los alumnos del $5^{\circ}$ año de educación secundaria de la I. E. PNP José Héctor Rodríguez Trigoso-San Martin de Porres.

\section{Prueba de Hipótesis específica 4}

Ho No existe correlación significativa entre clima organizacional y las reacciones comportamentales en alumnos del $5^{\circ}$ año de educación secundaria de la I. E. PNP José Héctor Rodríguez Trigoso-San Martin de Porres.

H1 Existe correlación significativa entre clima organizacional y las reacciones comportamentales en alumnos del $5^{\circ}$ año de educación secundaria de la I. E. PNP José Héctor Rodríguez Trigoso-San Martin de Porres.

Tabla 15. Coeficiente de correlación de Spearman de la variable clima organizacional y la dimensión reacciones comportamentales.

\begin{tabular}{lccc}
\hline & & \multicolumn{2}{c}{$\begin{array}{c}\text { Reacciones } \\
\text { Comportamentales }\end{array}$} \\
\hline \multirow{2}{*}{ Rho de Spearman } & Clima organizacional & Coeficiente de correlación &,$- 254^{* *}$ \\
& & Sig. (bilateral) &, 007 \\
& & $\mathrm{~N}$ & 112 \\
\hline
\end{tabular}

**. La correlación es significativa al nivel 0,01 (bilateral). 
Los resultados del análisis estadístico en la Tabla 15 dieron cuenta de la existencia de una relación Rho de Spearman $r=-0,254$ entre la variable: clima organizacional y la dimensión reacciones comportamentales. Este grado de correlación indicó que la relación entre las variables fue inversa y tuvo un nivel de correlación baja. En consecuencia se pudo afirmar que existió relación inversa entre clima organizacional $\mathrm{y}$ las reacciones comportamentales en alumnos del $5^{\circ}$ año de educación secundaria de la I. E. PNP José Héctor Rodríguez Trigoso- San Martin de Porres.

\section{Decisión estadística}

La significación de $\mathrm{p}=0,007$ mostró que es menor a 0,5 lo que permitió señalar que la relación fue significativa, por lo tanto, se rechaza la hipótesis nula y se acepta la hipótesis alternativa, es decir, que el clima organizacional tuvo relación inversa y significativa en el estrés de los alumnos del $5^{\circ}$ año de educación secundaria de la I. E. PNP José Héctor Rodríguez Trigoso-San Martin de Porres.

\section{Prueba de Hipótesis específica 5}

Ho No existe correlación significativa entre clima organizacional y las estrategias de afrontamiento en alumnos del $5^{\circ}$ año de educación secundaria de la I. E. PNP José Héctor Rodríguez Trigoso-San Martin de Porres. (Tabla 16).

H1 Existe correlación significativa entre clima organizacional y las estrategias de afrontamiento en alumnos del $5^{\circ}$ año de educación secundaria de la I. E. PNP José Héctor Rodríguez Trigoso-San Martin de Porres. (Tabla 16).

Tabla 16. Coeficiente de correlación de Spearman de la variable clima organizacional y la dimensión estrategias de afrontamiento.

\begin{tabular}{lccc}
\hline & & $\begin{array}{c}\text { Estrategias de } \\
\text { Afrontamiento }\end{array}$ \\
\hline \multirow{3}{*}{ Rho de Spearman } & Clima organizacional & $\begin{array}{c}\text { Coeficiente de } \\
\text { correlación }\end{array}$ &,$- 224^{*}$ \\
& & Sig. (bilateral) &, 018 \\
& & $\mathrm{~N}$ & 112 \\
\hline
\end{tabular}

**. La correlación es significativa al nivel 0,05 (bilateral).

Los resultados del análisis estadístico en la Tabla 16 dan cuenta de la existencia de una relación Rho de Spearman $r=-0,224 *$ entre la variable: clima organizacional y la dimensión estrategias de afrontamiento. Este grado de correlación indica que la relación entre las variables es inversa y tiene un nivel de correlación baja. En consecuencia se puede afirmar que, existe relación inversa entre clima organizacional y las estrategias de afrontamiento en alumnos del $5^{\circ}$ año de educación secundaria de la I. E. PNP José Héctor Rodríguez Trigoso - San Martin de Porres. 
Gregorio Ernesto Tomás Quispe y Orlando Mamani Rodríguez

\section{Decisión estadística}

La significación de $\mathrm{p}=0,018$ mostró que es menor a 0,5 lo que permitió señalar que la relación fue significativa, por lo tanto, se rechaza la hipótesis nula y se acepta la hipótesis alternativa, es decir, que el clima organizacional tuvo relación inversa y significativa en el estrés de los alumnos del $5^{\circ}$ año de educación secundaria de la I. E. PNP José Héctor Rodríguez Trigoso-San Martin de Porres.

Luego de la estadística aplicada a los estudiantes, los resultados de la prueba de hipótesis general dan cuenta de la existencia de la relación Rho de Spearman $r=-0,367^{* *}$ entre las variables: clima organizacional y el estrés. Este grado de correlación indica que la relación entre las variables es inversa mostrándose un nivel de correlación baja. En consecuencia se puede afirmar que, existe relación negativa entre clima organizacional y estrés en alumnos del 5to. Año de educación secundaria de la I. E. PNP José Héctor Rodríguez Trigoso-San Martin de Porres. $(\mathrm{r}=$ $-0,367)$, esto indica que a un mal clima organizacional, el estrés es mayor.

Con respecto a la primera hipótesis específica las tablas describen el análisis estadístico donde dan cuenta de la existencia de una relación Rho de Spearman $r=-0,340^{* *}$ entre la variable: clima organizacional y la dimensión estresores. Este grado de correlación indica que la relación entre las variables es inversa y muestra una correlación baja. En consecuencia se puede afirmar que, existe relación inversa significativa entre clima organizacional y los estresores en alumnos del 5to. Año de educación secundaria de la I. E. PNP José Héctor Rodríguez Trigoso-San Martin de Porres, lo que implica que a mejor clima organizacional menor presencia de estrés en los alumnos.

Asimismo en la prueba de la segunda hipótesis específica los resultados del análisis estadístico describen la existencia de una relación Rho de Spearman $r=-0,387$ entre la variable: clima organizacional y la dimensión reacciones físicas. Este grado de correlación indica que la relación entre las variables es negativa y tiene un nivel de correlación baja. En consecuencia se puede afirmar que, existe relación negativa entre clima organizacional y las reacciones físicas en alumnos del 5to. Año de educación secundaria de la I. E. PNP José Héctor Rodríguez Trigoso-San Martin de Porres. Con respecto a la prueba de la tercera hipótesis específica los resultados del análisis estadístico dan cuenta de la existencia de una relación Rho de Spearman $r=-0,259$ entre la variable: clima organizacional y la dimensión reacciones psicológicas. Este grado de correlación indica que la relación entre las variables es negativa y tiene un nivel de correlación baja. En consecuencia se puede afirmar que, existe relación negativa entre clima organizacional $\mathrm{y}$ las reacciones psicológicas en alumnos del 5to. Año de educación secundaria de la I. E. PNP José Héctor Rodríguez Trigoso-San Martin de Porres. En la prueba de la cuarta hipótesis específica los resultados del análisis estadístico dan cuenta de la existencia de una relación Rho de Spearman $r=-0,254^{* *}$ entre la variable: clima organizacional y la dimensión reacciones comportamentales. Este grado de correlación indica que la relación entre las variables es inversa y tiene un nivel de correlación baja. En consecuencia se puede afirmar que, existe relación inversa entre clima organizacional $\mathrm{y}$ las reacciones comportamentales en alumnos del 5to. Año 
de educación secundaria de la I. E. PNP José Héctor Rodríguez Trigoso- San Martin de Porres. Finalmente en la prueba de la quinta hipótesis específica los resultados del análisis estadístico dan cuenta de la existencia de una relación Rho de Spearman $r=-0,224^{* *}$ entre la variable: clima organizacional y la dimensión estrategias de afrontamiento. Este grado de correlación indica que la relación entre las variables es negativa y tiene un nivel de correlación baja. En consecuencia se puede afirmar que, existe relación inversa entre clima organizacional y las estrategias de afrontamiento en alumnos del 5to. Año de educación secundaria de la I. E. PNP José Héctor Rodríguez Trigoso - San Martin de Porres.

\section{CONCLUSIONES}

En base a los estudios obtenidos, se pudo apreciar que el clima organizacional y el estrés están estrechamente relacionados, el estrés está presente en todos los medios y ambientes, incluido el educativo, en donde se centra el objetivo de esta discusión; de ahí que los psicólogos, los docentes y los padres de familia, hayan identificado la necesidad de conocer las implicaciones que tiene sobre el rendimiento de los estudiantes. Es importante que todos los involucrados estén dispuestos a enfrentar los cambios ocurridos dentro de esta nueva normalidad y poder aprender a confrontar situaciones que generen presión en el estudiante y así puedan controlar sus emociones sin llegar al límite de sus capacidades, convirtiendo estas situaciones en estrés que a corto o largo plazo pueda generarles daños en su salud tanto física como mental.

\section{REFERENCIAS}

Cisneros, M., \& Paredes, W. (2013). Análisis de los Estilos de Liderazgo y su impacto en el clima organizacional de la empresa Pública de Petróleos en el Ecuador. Quito, Ecuador: Trabajo especal de grado de la Universidad Politécnica Salesiana para optar al título de Magister en Administración de empresas

Hernández, R., Fernández, C., \& Baptista, P. (2010). Metodología de la Investigación. Mc Graw Hill Interamericana

Loaiza, E., Salazar, P., Espinoza, L., \& Lozano, M. (2019). Clima Organizacional en la Administración de empresas: Un enfoque de género. Revista Científca Mundo de la Investigación y el Conocimiento 3(1), 3-25

Osorio, J. E., \& Cárdenas, L. (2017). Estrés laboral: estudio de revisión. . Diversitas, 13(1), 81-90

Palella Stracuzzi, S., \& Martins Pestana, F. (2010). Metodología de la investigación cuantitativa. Caracas, Venezuela: FEDUPEL, Fondo Editorial de la Universidad Pedagógica Experimental Libertador

Quito, J. V., Tamayo, M., Buñay, D., \& Neira, 0. (2017). Estrés académico en estudiantes de tercero de bachillerato de unidades educativas particulares del Ecuador. Revista electrónica de psicología Iztacala, 20(3) , 253-276

Segredo P, A. (2013). Clima organizacional en la gestión del cambio para el desarrollo de la organización. Revista Cubana de Salud Pública, 39(2), 385-393.

Williams, L. (2013). Estudio Diagnóstico de clima laboral en una dependencia pública. Monterrey, Nuevo León, México: Trabajo especial de grado de la Universidad Autónoma de Nuevo León para optar al título de Magister en Psicología con orientación laboral y organizacional 